\title{
Three-Way Decomposition and Nuclear Magnetic Resonance
}

\author{
Martin Billeter ${ }^{1}$ and Vladislav Orekhov ${ }^{2}$ \\ ${ }^{1}$ Biochemistry and Biophysics, Göteborg University, Box 462, 40530 Göteborg, Sweden \\ martin.billeter@bcbp.gu.se \\ ${ }^{2}$ Swedish NMR Centre at Göteborg University, Box 462, 40530 Göteborg, Sweden \\ orov@nmr.se
}

\begin{abstract}
Nuclear Magnetic Resonance (NMR) is a widely used tool in functional and structural genomics for the study of three-dimensional structures of proteins. The experimental data obtained by this method are multidimensional spectra consisting of about $10^{7}$ data points. We demonstrate that Three Way Decomposition (TWD) provides an inherently suitable tool for the analysis of these spectra. We apply here TWD for the first time to a NOESY-NOESY spectrum, which in terms of number of signals is among the most complex spectra. The application shows that the three-dimensional NMR spectra can be faithfully described by the components resulting from TWD, yielding among other advantages a data compression factor of over 100. The inherent relation between NMR and TWD is demonstrated on the NOESY-NOESY spectrum by deducing the TWD model from the mathematical description of the NMR experiment. Applicability of TWD to various types of NMR spectra, the use of sparse experimental data sets in order to reduce instrument time and other aspects of the analysis are discussed.
\end{abstract}

\section{Introduction}

The current contribution concerns the processing and analysis of large data sets $\left(\sim 10^{7}\right.$ individual measurements) that arise from nuclear magnetic resonance (NMR [1]) experiments when applied to biological macromolecules, in particular proteins. The importance of the problem can be illustrated on the one hand by the interest in deciphering the human genome, and on the other hand by the large NMR investments of pharmaceutical companies for the development of new medicines. The algorithm proposed here is three-way decomposition (TWD), which has an inherent relation to multidimensional NMR data sets as shown below. The TWD algorithm has been presented earlier as such [2] and more recently as a tool for the analysis of NMR spectra [3-5]. Here, we complement earlier, in part more technical publications [6] by demonstrating the application of TWD to the most complex NMR spectrum so far (in terms of number of signals). In the rest of this introduction, some general aspects of proteins and of NMR are mentioned.

Proteins and DNA are very large molecules (macromolecules) that form the basis of life. DNA molecules serve mostly as medium for information storage, and worldwide efforts to read the entire genomic information are currently being completed. 
Genes from the most important part of this information: they encode the building plans for proteins. Proteins play a crucial role in almost any metabolic process, and the study of the function of these gene products is referred to as "functional genomics" and enjoys at present enormous attention. Typically, proteins exert their function by intermolecular interactions, and thus an understanding of the function of a protein requires knowledge of its three-dimensional (3D) structure. In addition, the description of the internal dynamics of these molecules may often provide significant clues when explaining function at a molecular level. Besides fundamental questions about life, numerous practical applications rely on knowledge of structure and dynamics of proteins, an example being the design of clinically active reagents ("drug discovery").

Proteins are molecules that consist of thousands of atoms. Experimental methods for 3D structure determinations that yield coordinates for each atom must therefore provide a large amount of data. NMR is one of few methods that presently can provide complete structures of macromolecules at atomic resolution, and it is the prime experimental method for the characterization of internal molecular dynamics. NMR provides multidimensional spectra with typical sizes of several million data points. Their analysis offers a significant computational challenge. This is further accentuated by a variety of experimental artifacts, by limited access to the expensive NMR equipment and by problems related to the availability of sufficient and stable protein samples.

The purpose of this contribution is twofold. TWD, an algorithm for the analysis of three- or higher dimensional matrices is applied to a NMR spectrum of type NOESYNOESY [7]. This application allows a relatively simple illustration of the intimate relation between 3D NMR spectroscopy and TWD by deducing the model assumption of TWD directly from the description of the NMR experiment. The discussion includes analyses of other types of NMR data sets, including spectra with information on 3D structure [3], on molecular dynamics [4] and on intermolecular binding as used in "drug discovery" [5]. It thus shows the wide applicability of TWD in the field of high-resolution NMR. Other issues addressed include spectrum reconstruction, data compression, and the handling of sparse data allowing significant savings of instrument time, or alternatively optimization of spectral resolution and sensitivity.

\section{Methods}

\subsection{Three-Way Decomposition}

Three-way decomposition (TWD) is a mathematical concept for the approximation of a three- or higher dimensional matrix by lower-dimensional matrices (often onedimensional) [2]. TWD has been introduced as a tool for data analysis in the early seventies under various names such as parallel factor analysis or canonical decomposition. Theoretical considerations concerned notably questions of uniqueness of optimal approximations and convergence behavior. Applications include data compression, chemometrics and more recently the processing of multidimensional NMR (nuclear magnetic resonance) spectra. While the use in chemometrics, e.g. the analysis of fluorescence data, concerns decomposition of matrices of about $10^{3}$ elements into less than ten components, data compression and NMR applications involves data 
matrices exceeding $10^{7}$ elements and requiring hundreds of components. TWD can be formulated as follows. Given a matrix $S$ with elements $s_{i j k}(i=1 . . I, j=1 . . J, k=1 . . K)$, find numbers $a^{\beta}$ and vectors $\boldsymbol{F} \mathbf{1}^{\beta}, \boldsymbol{F 2 ^ { \beta }}$ and $\boldsymbol{F} 3^{\beta}$ with elements $f 1_{i}^{\beta}, f 2_{j}^{\beta}$ and $f 3_{k}^{\beta}$, respectively, such that the norm

$$
\left|S-\Sigma_{\beta}\left(a^{\beta} \boldsymbol{F} \mathbf{1}^{\beta} \otimes \boldsymbol{F} 2^{\beta} \otimes \boldsymbol{F} 3^{\beta}\right)\right|^{2}
$$

becomes minimal.

The sum in this expression represents the fundamental model assumption of TWD: Direct products of one-dimensional vectors are sufficient to describe all features of a high-dimensional matrix. In the following we refer to $S$ as the (input) spectrum and to the entities in the sum over $\beta$ as amplitudes $a^{\beta}$ and shapes $\boldsymbol{F 1}^{\beta}, \boldsymbol{F}^{\beta}$ and $\boldsymbol{F 3}^{\beta}$, while the summation terms are called (output) components. The amplitudes $a^{\beta}$ result from the

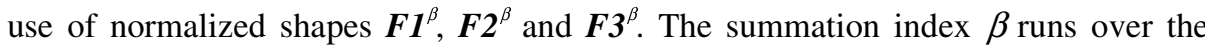
number of components used for the decomposition. The range for this index depends on the type of application. For typical 3D NMR spectra, which consist of several millions of data points, it is sufficient to use a few hundred components. Consequently, a description of the spectrum by components may yield a significant compression of the data. The redundancy present in many types of NMR spectra may thus be used to save experiment time when solving a modified problem [6]: Minimize

$$
\left|\boldsymbol{G} \bullet\left[\boldsymbol{S}^{\boldsymbol{Y}}-\Sigma_{\beta}\left(a^{\beta} \boldsymbol{F} \mathbf{1}^{\beta} \otimes \boldsymbol{F} \mathbf{2}^{\beta} \otimes \boldsymbol{F} \mathbf{3}^{\beta}\right)\right]\right|^{2}
$$

where the matrix $\boldsymbol{G}$ contains elements $g_{i j k} \in\{0,1\}$ that indicate the absence or presence of a data point in $S^{\prime}$, and $\bullet$ describes element-wise multiplication of matrices.

The product $\boldsymbol{G} \bullet \boldsymbol{S}$ ' is used to denote a sparse matrix $\boldsymbol{S}$ as input for the decomposition. Note that while the input sparse data matrix $S$ lacks many entries, the shapes $\boldsymbol{F 1 ^ { \beta } , \boldsymbol { F 2 }}{ }^{\beta}$ and $\boldsymbol{F 3}^{\beta}$ representing the output of TWD are complete, allowing to reconstruct a full matrix description. As shown later, omission of elements of $\boldsymbol{S}$ results in savings of NMR experiment time, which is a relevant issue when considering the price of instruments (several million dollars) and the duration of experiments (for practical reasons usually limited to about one week). An alternative formulation of the gains achieved with TWD is that with a given total experiment time one may improve spectral resolution. Furthermore, compared to conventional methods based on Fourier transform, which requires uniform data sampling, the use of sparse data matrices allows for optimized sampling and provides improved spectral sensitivity.

\subsection{Nuclear Magnetic Resonance}

NMR is based on the interaction of spins with magnetic fields ${ }^{1}$. Avoiding physical explanations as much as possible, the following description concentrates on aspects required below when showing the intimate relation between 3D NMR and TWD using NOESY-NOESY spectra [7]. We need only consider the spins of the nuclei of hydrogen atoms (i.e. simple protons). A typical protein contains a few hundred hydrogen atoms and thus a large number of probes for NMR measurements. In a strong, static magnetic field, spins assume preferred states (orientations), corresponding to different magnetizations. An analogy of this effect is the alignment of a compass needle in the magnetic field of the earth. In a NMR experiment, short pulses of electromagnetic ir- 
radiation can manipulate the spin magnetization. For example, pulses can be designed to flip the magnetization from an initial state parallel to the strong static magnetic field to a perpendicular orientation. Magnetization perpendicular to the magnetic field will precess around the field direction. A third effect that we need to mention is the exchange of magnetization between nearby spins. This transfer occurs only across short distances $(<0.5 \mathrm{~nm})$. It is popular to view NMR experiments as a series of steps called a "pulse sequence", and the individual steps can be characterized by a set of "recipes" [1]. The following is a simplified and incomplete list of recipes, reduced to meet our purposes. Spin magnetization is described by a vector $\boldsymbol{I}$.

(a) The initial (equilibrium) orientation of all spin magnetization is along the static magnetic field, which defines the direction of the z-axis. Thus only the components $I z$ are different from zero.

(b) A "90x-pulse" rotates the magnetization by $90^{\circ}$ around the $\mathrm{x}$-axis: " $I z \rightarrow I y$ ".

(c) Precession with time $t$ of magnetization perpendicular to the z-axis occurs with a frequency $\Omega_{l}$ characteristic for each spin: "Iy $\rightarrow I y \cos \left(\Omega_{t} t\right)+I x \sin \left(\Omega_{t} t\right)$ ".

(d) Different spins (labeled $\alpha$ and $\beta$ ) oriented along the z-axis and separated by a short distance interact with each other with a mixing efficiency $m_{\alpha \beta}: " I z^{\alpha} \rightarrow$ $m_{\alpha \beta} z^{\beta, "}$.

(e) Observation along the y-axis yields a scalar (amplitude), $a$, as the sum of contributions from all spins: " $\Sigma \gamma I^{\gamma} \rightarrow a$ ", where $\gamma$ enumerates all spins.

In a 3D NMR experiment, successive magnetization transfers between three different spins $\boldsymbol{I}, \boldsymbol{J}$ and $\boldsymbol{K}$ provide a signal that carries information on all three characteristic frequencies $\Omega_{p}, \Omega_{j}$ and $\Omega_{K}$. This signal can be reported in a 3D spectrum at the position given by the coordinate triple $\left(\Omega_{p}, \Omega_{j}, \Omega_{K}\right)$. The complete spectrum will include signals for all possible magnetization pathways, i.e. for all spin triples for which the transfer defined by the experiment takes place.

\subsection{Experiments}

A three-dimensional NOESY-NOESY ${ }^{7}$ experiment was collected at $30^{\circ} \mathrm{C}$ on a sample with the protein ubiquitin $(1 \mathrm{mM}$ in $50 \mathrm{mM}$ potassium phosphate buffer $\mathrm{pH} 5.8$, $\mathrm{H} 2 \mathrm{O} / \mathrm{D} 2 \mathrm{O}$ 9:1). The experiment required 144 hours on a $600 \mathrm{MHz}$ Varian Unity Inova spectrometer (mixing times: $100 \mathrm{~ms}$ for both NOESY steps). The experimental data formed a matrix of $120 * 180 * 608$ complex data points (sizes in the dimensions $\mathrm{t} 1, \mathrm{t} 2$ and $\mathrm{t} 3$ ). The spectrum was Fourier transformed in all dimensions yielding a data matrix with $240 * 810 * 901$ entries. For an illustration, a section of this spectrum with matrix size $240 * 28 * 901$ was selected (range $8.75-8.5 \mathrm{ppm}$ in the second dimension), and this sub-spectrum was subjected to TWD using 27 components.

\section{Results}

\subsection{Application to NOESY-NOESY Spectra}

The NOESY-NOESY sub-spectrum described in Methods was chosen as input to TWD; a plane perpendicular to the second dimension is shown in Fig. 1 (left side). 
Crowded groups of signals along the horizontal and vertical axes correspond to diagonal planes in the 3D matrix. Genuine 3D peaks connecting three different hydrogen atoms, and carrying structural information, are located outside any diagonal planes. The vertical stripe of peaks visible in the middle of the spectrum is an artifact caused by the strong signal of water. The main result of the decomposition with 27 components is shown in Fig. 1 (right side), where a reconstruction of the plane from the left side is presented. The reconstruction corresponds to the sum of all 27 components according to expression (1). All important features of the original spectrum, including strong and weak signals, are preserved in the reconstruction, thus illustrating the good fit of the model given by expression (1) to the experiment. Note that the number of used components is several times smaller than the number of peaks in the sub-spectrum, which totally contains 28 planes like the one shown in Fig. 1. This justifies the validity of the model and proves the redundancy of the experimental data. A measure of the latter is given by the compression factor. The original data consisting of $240 * 28 * 901$ data points is approximated by the $(240+28+901) * 27$ parameters of the model. The compression factor, given by the ratio of the two values, is as high as 185. This compression can be used directly to simplify handling of data set of hundreds of megabytes. Alternatively, redundancy of the data can be used to save experiment time by applying sparse detection.

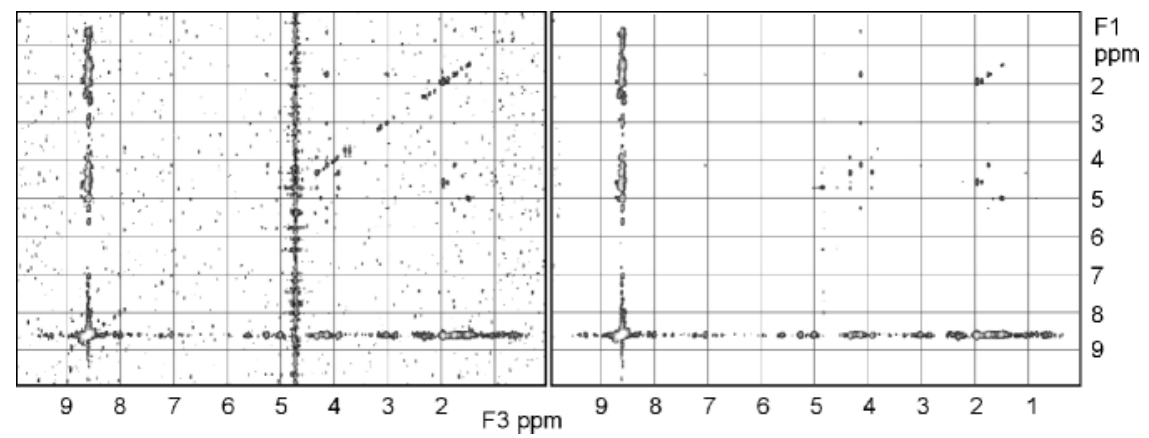

Fig. 1. Selected plane along the F1 and F3 dimensions from a 3D NOESY-NOESY spectrum (left side). The strong vertical line of signals in the middle is an artifact caused by strong signals from water. For the decomposition, a stripe covering this artifact was bleached out. Other strong signals along lines describe diagonal planes of the 3D spectrum. Genuine cross peaks defined by frequencies from three hydrogen nuclei lie off these diagonals, together with weaker noise peaks. A sub-spectrum with 28 planes (including the one shown here) was decomposed using 27 components. Reconstruction of this sub-spectrum by summing the resulting components according to expression (1) yields for the selected plane the result shown on the right side. In addition to significant data compression, noise is strongly suppressed.

\subsection{Relation between 3D NMR and TWD}

TWD and 3D NMR spectroscopy are intimately related, as can be shown by deducing the model assumption for TWD (expression (1)) from the description of 3D NMR experiments. This connection can be shown on a general level; however, in the following we use for this purpose the experiment presented above, NOESY-NOESY. Fig. 
2A schematically summarizes this experiment. Initially, all hydrogen nuclei are irradiated; these nuclei are referred to as spins $I$ and enumerated with the index $\alpha$. Magnetization is then transferred to neighboring nuclei, called $\boldsymbol{J}$ with the index $\beta$, provided that the distance $\boldsymbol{I}^{\alpha}$ to $\boldsymbol{J}^{\beta}$ is short. A second transfer step brings the magnetization to neighbors of $\boldsymbol{J}$, referred to as nuclei $\boldsymbol{K}$ with the index $\gamma$. The individual steps of the experiment together with description of the resulting spin states and the "recipes" used for the steps (see Methods) are listed in Table 1. The initial states with magnetization vectors parallel to the magnetic field, $I z^{\alpha}$, are rotated to the y-axis by the first pulse. During a time $t 1$ the spins process around the z-axis, so that their components along the $\mathrm{y}$-axis are given by $\cos \left(\Omega_{1}^{\alpha} t 1\right)$. After a further pulse, the magnetization can be transferred to neighboring spins $\boldsymbol{J}^{\beta}$ in a step called "mixing". Since this transfer occurs only over short distances, the sum over $\alpha$ is reduced to a small number of terms where the transfer efficiency $m_{\alpha \beta}$ differs significantly from zero. We can abbreviate the sum over $\alpha$ as a function $F 1^{\beta}$; note that this function is unique for each spin $\boldsymbol{J}^{\beta}$, and it depends on the precession time $t 1$. Repeating the sequence pulseprecession-pulse-mixing we have labeled the magnetization in addition with the frequency of the nucleus $\boldsymbol{J}^{\beta}$, and transferred the magnetization to nuclei referred to as $\boldsymbol{K}^{\gamma}$. The short notation $F 2^{\beta}$ for $\cos \left(\Omega_{J}^{\beta} t 2\right)$ cannot include the sum over $\beta$ since $F 1^{\beta}$ depends as well on this summation index. $F 2^{\beta}$ is a function of the precession time $t 2$. A final pulse-precession combination labels the magnetization with the frequencies of the nuclei $\boldsymbol{K}^{\gamma}$. The observation, which yields a scalar given by an amplitude $a^{\beta}$, is the sum of all existing magnetization. Thus, we can define a third function $F 3^{\beta}$ that collects the terms in the summation over $\gamma$, and that depends on $t 3$.
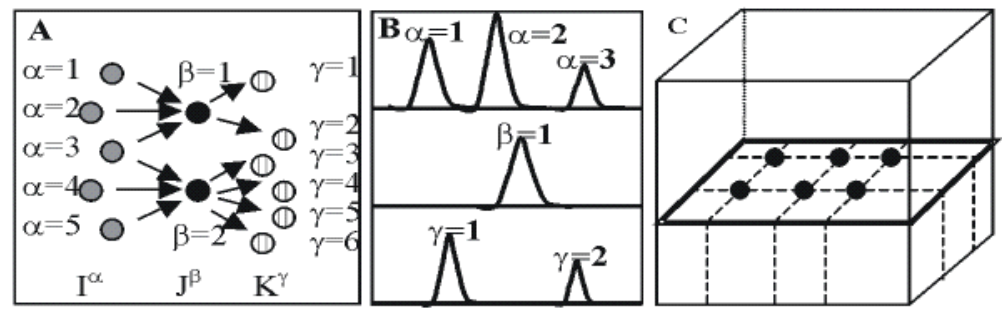

Fig. 2. (A) Schematic summary of the NOESY-NOESY experiment. Spheres denote individual spins with different shading for the spins $\boldsymbol{I}, \boldsymbol{J}$ and $\boldsymbol{K}$ (see text) and Greek lettering for their enumeration. Arrows indicate magnetization transfers. (B) Fourier transformed shapes corresponding to one component, $\boldsymbol{J}^{1}$, and $\boldsymbol{I}$ and $\boldsymbol{K}$ spins that interact with it. (C) Schematic result of the direct product of the Fourier transformed shapes from (B) according to expression (1). 
Table 1. NOESY-NOESY experiment and connection to TWD

\begin{tabular}{|c|c|c|c|}
\hline Step & Spin state description $^{1}$ & $\begin{array}{l}\text { Rec- } \\
\text { ipe }^{2}\end{array}$ & Comments \\
\hline initial state & $\overline{\sum_{\alpha} \mathrm{Iz}^{\alpha}}$ & (a) & $\bar{\alpha} \alpha$ enumerates hydrogens \\
\hline 90x-pulse & $\Sigma_{\alpha} \mathrm{Iy}^{\alpha}$ & (b) & $90^{\circ}$ rotation around $\mathrm{x}$-axis \\
\hline t1-evolution & $\Sigma_{\alpha} \mathrm{Iy}^{\alpha} \cos \left(\Omega_{1}^{\alpha} \mathrm{t} 1\right)$ & (c) & precession during $\mathrm{t} 1^{3}$ \\
\hline 90x-pulse & $-\Sigma_{\alpha} \mathrm{Iz} \mathrm{z}^{\alpha} \cos \left(\Omega_{1}^{\alpha} \mathrm{t} 1\right)$ & (b) & $90^{\circ}$ rotation around $\mathrm{x}$-axis \\
\hline mixing & $-\sum_{\beta} \mathrm{Jz}^{\beta} \sum_{\alpha} \mathrm{m}_{\alpha \beta} \cos \left(\Omega_{1}^{\alpha} \mathrm{t} 1\right)$ & (d) & short distance interaction \\
\hline 90x-pulse & $-\Sigma_{\beta} \mathrm{Jy}{ }^{\beta} \mathrm{F} 1^{\beta}$ & (b) & $\begin{array}{l}90^{\circ} \text { rotation around } \mathrm{x} \text {-axis } \\
\text { define } \mathrm{F} 1^{\beta}=\Sigma_{\alpha} \mathrm{m}_{\alpha \beta} \cos \left(\Omega_{1}^{\alpha} \mathrm{t} 1\right)\end{array}$ \\
\hline t2-evolution & $-\Sigma_{\beta} \mathrm{Jy}^{\beta} \cos \left(\Omega_{\mathrm{J}}^{\beta} \mathrm{t} 2\right) \mathrm{F} 1^{\beta}$ & (c) & precession during $\mathrm{t}^{3}$ \\
\hline 90x-pulse & $\Sigma_{\beta} \mathrm{Jz} \mathrm{z}^{\beta} \mathrm{F} 2^{\beta} \mathrm{F} 1^{\beta}$ & (b) & $\begin{array}{l}90^{\circ} \text { rotation around } \mathrm{x} \text {-axis } \\
\text { define } \mathrm{F} 2^{\beta}=\cos \left(\Omega_{\mathrm{J}}^{\beta} \mathrm{t} 2\right)\end{array}$ \\
\hline mixing & $\sum_{\beta} \sum_{\gamma} \mathrm{Kz}^{\gamma} \mathrm{m}_{\gamma \beta} \mathrm{F} 2^{\beta} \mathrm{F} 1^{\beta}$ & (d) & short distance interaction \\
\hline 90x-pulse & $\Sigma_{\beta} \Sigma_{\gamma} \mathrm{Ky}^{\gamma} \mathrm{m}_{\gamma \beta} \mathrm{F} 2^{\beta} \mathrm{F} 1^{\beta}$ & (b) & $90^{\circ}$ rotation around $\mathrm{x}$-axis \\
\hline t3-evolution & $\Sigma_{\beta} \sum_{\gamma} \mathrm{Ky}^{\gamma} \mathrm{m}_{\gamma \beta} \cos \left(\Omega_{\mathrm{K}}^{\gamma} \mathrm{t} 3\right) \mathrm{F} 2^{\beta} \mathrm{F} 1^{\beta}$ & (c) & precession during $\mathrm{t} 3^{3}$ \\
\hline observation & $\Sigma_{\beta} \mathrm{a}^{\beta} \mathrm{F} 3^{\beta} \mathrm{F} 2^{\beta} \mathrm{F} 1^{\beta}$ & (e) & define $F 3^{\beta}=\sum_{\gamma} m_{\gamma \beta} \cos \left(\Omega_{K}^{\gamma}{ }^{\gamma} 3\right)$ \\
\hline
\end{tabular}

1 In the present simplified explanation of an experiment, terms are dropped after some of the steps; these terms are not essential for the outcome of the resulting spectrum.

2 See Methods for a list of the recipes used.

3 Terms in $\sin (\Omega \mathrm{t})$ are not affected by the following step and therefore not further considered.

The final data recorded forms a matrix with the three axes given by the precession times $t 1, t 2$ and $t 3$. To this end, discrete values for these three precession times are selected. The pulse sequence of Table 1 is repeated for all combinations of selected $t 1$ and $t 2$ values. At the end of each pulse sequence, observation occurs for all selectedvalues of $t 3$. With the resulting $3 \mathrm{D}$ grid of observations, the functions $F 1^{\beta}, F 2^{\beta}$ and $F 3^{\beta}$ become vectors that are equivalent to the shapes used in expression (1). Components are enumerated by the index $\beta$, and each component corresponds to one spin $\boldsymbol{J}^{\beta}$. The shapes consist of a (discrete) cosine function with the characteristic frequency $\Omega_{j}^{\beta}$ in $F 2^{\beta}$, and a sum of cosines for the characteristic frequencies of the spins $I^{\alpha}$ in $F I^{\alpha}$ and $\boldsymbol{K}^{\gamma}$ in $F 3^{\gamma}$ that interact with the spin $\boldsymbol{J}^{\beta}$ as follows:

$$
\begin{gathered}
F 1^{\beta}(t 1)=\Sigma_{\alpha} m_{\alpha \beta} \cos \left(\Omega_{l}^{\alpha} t 1\right) \\
F 2^{\beta}(t 2)=\cos \left(\Omega_{J}^{\beta} t 2\right) \\
F 3^{\beta}(t 3)=\Sigma_{\gamma} m_{\gamma \beta} \cos \left(\Omega_{K}^{\gamma} t 3\right)
\end{gathered}
$$


Fourier transform of these three discrete functions yields the 1D-spectra of Fig. 2B (for one of the $\beta$-nuclei of Fig. 2A). The direct product of these three 1D-spectra provides the 3D spectrum of Fig. 2C, and summation over the 3D spectra of all nuclei $\boldsymbol{J}^{\beta}$ corresponds to the experimental data after Fourier transform in all three dimensions according to expression (1). Analysis of a 3D NMR data set by TWD thus provides (a) a way of data compression and (b) a separation of the subset of signals that belong to individual nuclei $\boldsymbol{J}^{\beta}$.

\section{Discussion}

\subsection{Applicability of TWD to NMR Data of Proteins}

The above derivation of the model expression (1) for TWD for the NOESY-NOESY illustrates for a particular 3D NMR experiment the close relation between NMR data sets and TWD. Other examples are heteronuclear NMR experiments that rely on additional nuclei with spins $1 / 2$, namely the isotopes carbon-13 $\left({ }^{13} \mathrm{C}\right)$ and/or nitrogen-15 $\left({ }^{15} \mathrm{~N}\right)$. Thus, the application of TWD to 3D NMR spectra was first demonstrated for another experiment, a ${ }^{15} \mathrm{~N}-\mathrm{NOESY}-\mathrm{HSQC}^{3}$. The connection between NMR and TWD is, however, much more general; in fact it holds also for data sets that are not strictly the result of 3D NMR experiments. Since no requirement is made on the form of the shapes $\boldsymbol{F} 1^{\beta}, \boldsymbol{F} 2^{\beta}$ and $\boldsymbol{F 3}^{\beta}$ of expression (1), one may consider any type of modulation. Two such examples have been presented earlier [4,5]; both rely on the same simple two-dimensional NMR experiment yielding a ${ }^{15} \mathrm{~N}$-HSQC spectrum. In this spectrum, every hydrogen-nitrogen pair connected by a single chemical bond gives rise to a signal defined by the characteristic frequencies of the two nuclei, and these frequencies define the shapes $\boldsymbol{F} \mathbf{1}^{\beta}$ and $\boldsymbol{F} 2^{\beta}$. At a first glance this may look little informative; however, the usefulness of these spectra becomes apparent when considering a series of such spectra with a particular modulation. Thus, one may record ${ }^{15} \mathrm{~N}-\mathrm{HSQC}$ spectra where the signal intensity is modulated by an additional parameter that describes relaxation. The third function, $\boldsymbol{F 3}^{\beta}$, follows an exponential expression, and curve fitting yields a decay time for each hydrogen-nitrogen pair, i.e. a dense characterization of the different relaxation behavior over the protein [4]. Relaxation data in turn provide information on the internal molecular dynamics and thus allow a better description of the biological function. A second application of ${ }^{15} \mathrm{~N}-\mathrm{HSQC}$ spectra concerns ligand binding to a protein. In "drug discovery", the goal is to detect ligands with good binding properties to a given target protein. These ligands (or "leads") may after further improvement become a drug with a selective clinical activity that targets the given protein. For such a search, libraries with sometimes 100'000 small molecules are screened. Although several ligands may be tested simultaneously, the large number of spectra recorded requires some automated and reliable high-throughput screening method. The spectral feature to recognize binding in a given mixture is change of position of the signals from those hydrogen-nitrogen pairs that are part of the binding site. With TWD applied to a set of ${ }^{15} \mathrm{~N}$-HSQC spectra recorded for different mixture of the target protein and potential ligands, the third function, $\boldsymbol{F 3}^{\beta}$, simply indicates the presence or absence of a signal for the hydrogen-nitrogen pair $\beta$. For a given signal, the function adopts a non-zero value for all spectra where no binding oc- 
curs near the corresponding atom pair, and drops to zero when the signal is shifted away due to ligand binding. (Other components may describe the complementary behavior for signals at the new position.)

\subsection{Sparse Data Sets}

So far the discussion was limited to the description of a complete, large 3D matrix with experimental NMR data by a sum of direct products of $1 \mathrm{D}$ vectors. This resulted in several advantages. First, the use of lower rank matrices yields a significant compression of the data, which due to the tight relation between the NMR data and the TWD model can be achieved without relevant loss of accuracy. The second advantage is a consequence of the NMR-TWD relation. The decomposition produces a natural separation of the NMR data, i.e. the individual terms of the sum in expression (1) typically correspond to individual nuclei and the shapes $\boldsymbol{F 1 ^ { \beta }}, \boldsymbol{F 2 ^ { \beta }}$ and $\boldsymbol{F 3}^{\beta}$ describe interactions of these nuclei.

A further advantage was very recently suggested ${ }^{8}$; it is based on expression (2) and concerns incomplete experimental data. Significant savings of example 75\% in NMR instrument time can be achieved. The processing of the $25 \%$ experimental data by the algorithm corresponding to expression (2) provides complete shapes $\boldsymbol{F 1 ^ { \beta }}, \boldsymbol{F 2}^{\beta}$ and $\boldsymbol{F 3}^{\beta}$ for all components. Multiplication of these functions and addition as in expressions (1) and (2) subsequently yields the reconstruction of a complete spectrum. Tests show that the reconstructed spectrum closely corresponds to a reference spectrum that would result from the recording of a full NMR data set.

\subsection{Mixing and Regularization}

The decomposition according to expression (1) is for data sets with a dimensionality exceeding three in general unique as long as the shapes differ in all three dimensions. If signals overlap in one dimension, a phenomenon referred to as mixing occurs (this mixing has nothing to do with the mixing mentioned in Table 1). Both the appearances of mixing and automated procedures for removing it have been described in detail $[3,9]$. In short, a mixed component contains signal intensity from two or more components. With additional information, e.g. that signals must be strictly nonnegative, one can determine a transformation that "demixes" such components. Consider for example two mixed components with near-identical shapes $\boldsymbol{F 3}^{\beta}$. The following transformations yield non-mixed components $\boldsymbol{F} \mathbf{1}^{1} \cos (\phi)+\boldsymbol{F} \mathbf{1}^{2} \sin (\psi)$ and $\boldsymbol{F} \mathbf{2}^{i}$ $\cos (\phi)+\boldsymbol{F} 2^{2} \sin (\psi)$. Signals that overlap in two dimensions can be combined into a single component. Sometimes, due to non-perfect appearance of the experimental data with respect to the model of expression (1), two components result from TWD that describe essentially only one component. Often, these two components have very large amplitudes with different signs such that they mostly cancel each other. To avoid this phenomenon, a Tikhonov regularization term is introduced that penalizes amplitudes that are significantly larger than the others [10]. The term, which is added to expression (1), has the following form $R \Sigma_{\beta}\left(a^{\beta}\right)^{2}$, where $\mathrm{R}$ is the regularization factor. 


\section{Conclusions}

TWD is a very versatile tool for the processing and analysis of NMR experiments. It has an intimate relation to 3D NMR spectra, and it is applicable to many different types of NMR data. Besides aspects that are specific to individual NMR data sets, e.g. grouping of signals that involve one particular nucleus as in the example of Table 1, TWD offers two major advantages. First, the large NMR spectra with typically several million data points can be efficiently compressed while maintaining high similarity to the original data. Second, the original data, but also selected subsets thereof, can be reconstructed by multiplying shapes and summing components according to expression (1). These reconstructions allow visualization and analysis with the normal tools used for NMR spectra. A special case relying on reconstruction is the decomposition of sparsely recorded data sets, saving costly instrument time, which yields complete shapes and thus allows reconstruction of a full data matrix.

Acknowledgements. This work was supported by grants 621-2001-3095 and 6212001-3014 from the Swedish Research Council. The authors would like to thank Ilghiz Ibragimov for fruitful discussions. The NMR experiment was performed at the Swedish NMR Centre.

\section{References}

1. Levitt, M.H.: Spin Dynamics. Wiley, New York (2001)

2. Kruskal, J. B.: Three-way arrays: rank and uniqueness of trilinear decomposition, with application to arithmetic complexity and statistics. Linear Algebra Appl. 18 (1977) 95-138

3. Orekhov, V., Ibraghimov, I.V., Billeter, M.: MUNIN: a new approach to multidimensional NMR spectra interpretation. J. Biomol. NMR 20 (2001) 49-60

4. Korzhnev, D. M., Ibraghimov, I. V., Billeter, M., Orekhov, V.: MUNIN: application of three-way decomposition to the analysis of heteronuclear NMR relaxation data. J. Biomol. NMR 21 (2001) 263-268

5. Damberg, C. S., Orekhov, V., Billeter, M.: Automated analysis of large sets of heteonuclear correlation spectra in NMR-based drug design. J. Med. Chem. (2002) in press

6. Ibraghimov, I.: Application of the three-way decomposition for matrix compression. Numer. Linear Algebra Appl. 9 (2002) 551-565

7. Boelens, R., Vuister, G.W., Koning, T.M.G., Kaptein R. Observation of spin duffusion in biomolecules by three.dimensional NOE-NOE Spectroscopy. J. Am. Chem. Soc. 111 (1989) 8525-8526

8. Orekhov, V., Ibraghimov, I, Billeter, M.: Optimizing resolution in multidimensional NMR by three-way decomposition. Submitted

9. Gutmanas, A., Jarvoll, P., Orekhov, V., Billeter, M.:Three-way decomposition of a complete 3D 15N-NOESY-HSQC. J. Biomol. NMR. 24 (2002) 191-201

10. Tikhonov, A.N., Samarskij, A.A.: Equations of mathematical physics. Dover, New York (1990) 